\title{
AN INVENTORY OF THE MOTH FAUNA (LEPIDOPTERA) OF JATINGA, ASSAM, INDIA
}

H.S. Rose

Department of Zoology, Punjabi University, Patiala, Punjab 147002, India

\begin{abstract}
An inventory of the moth fauna of a tiny North Eastern locality, Jatinga in North Cachar Hills (Haflong: Assam: latitude $24^{\circ}$ to $28^{0} 18^{\prime} \mathrm{N}$, longitude $89^{\circ} 46^{\prime} \mathrm{E}$ to $97^{\circ} 4^{\prime} \mathrm{E}$ ) is prepared for the first time. It includes 81 species referable to the genera Aetholix Lederer, Agathodes Guenée, Agrotera Schrank, Arthroschista Hampson, Bocchoris Moore, Botyodes Guenée, Chalcidoptera Butler, Diaphania Hübner, Dichocrocis Lederer, Glyphodes Guenée, Hemopsis Kirti and Rose, Herpetogramma Lederer, Heterocnephes Lederer, Hymenia Hübner, Indogrammodes Kirti and Rose, Ischnurges Lederer, Lamprosema Hübner, Limbobotys Munroe, Lygropia Lederer, Maruca Walker, Meroctena Lederer, Nagiella Munroe, Nausinoe Hübner, Nevrina Guenée, Notarcha Meyrick, Pagyda Walker, Palpita Hübner, Patania Moore, Phostria Hübner, Pionea Guenée, Polythlipta Lederer, Pramadea Moore, Prophantis Warren, Prorodes Swinhoe, Pycnarmon Lederer, Rhagoba Moore, Samea Guenée, Satanastra Meyrick, Sisyrophora Lederer, Syllepte Hübner, Synclera Lederer, Tangla Swinhoe, Tetridia Warren and Toxobotys Munroe and Mutuura of the subfamily Pyraustinae; 39 species belonging to the genera Achaea Hübner, Alamis Guenée, Anisoneura Guenée, Anua Walker, Artena Walker, Caranilla Moore, Chalciope Hübner, Cocytodes Guenée, Dysgonia Hübner, Entomogramma Guenée, Ercheia Walker, Eupatala Ragonot, Grammodes Guenée, Hypopyra Guenée, Lagoptera Guenée, Lygniodes Guenée, Mocis Hübner, Ophisma Ochsenheimer, Parallelia Hübner, Pericyma HerrichSchaffer, Phyllodes Boisduval, Pindara Moore, Thyas Hübner and Trigonodes Guenée of the subfamily Catocalinae; 44 species pertaining to the genera Aedia Hübner, Amphigonia Guenée, Anereuthina Hübner, Anomis Hübner, Asta Walker, Bamra Moore, Calyptra Ochsenheimer, Chrysopera Hampson, Delgamma
\end{abstract}

Moore, Episparina Berio, Episparis Walker, Ericeia Walker, Eudocima Billberg, Fodina Guenée, Hamodes Guenée, Hulodes Guenée, Hypocala Guenée, Hypospila Guenée, Ischyja Hübner, Koptoplax Hampson, Lacera Guenée, Ommatophora Guenée, Oraesia Guenée, Othreis Hübner, Oxyodes Guenée, Platyja Hübner, Ramadasa Moore, Rhytia Hübner, Serrodes Guenée, Speiredonia Hübner, Sympis Guenée and Trichopolydesma Berio of the subfamily Ophiderinae; and 16 species belonging to the genera Acronicta Oschsenheimer, Apsarasa Moore, Athetis Hübner, Callopistria Hübner, Callyna Guenée, Chasmina Walker, Craniophora Snellen, Dipterygina Sugi, Eutamsia Fletcher, Prospalta Walker, Spodoptera Guenée and Yepcalphis Nye of the subfamily Acronictinae. In all, 180 species have been recorded from this locality, which is situated in one of the biodiversity hot spots in India. It has been made out that the spot be given due attention both by the Entomologists and Ornithologists. The fruit piercing for juice sucking and skin piercing for blood sucking behaviour of certain Ophiderinae taxa have been quoted to attract the attention of Lepidopterists in India. It has also been emphasized that the life history studies and location of the larval host plants of non-pest species are essential from the conservation point of view.

\section{Keywords}

Pyraustinae, Catocalinae, Ophiderinae, Acronictinae, Lepidoptera, moth, biodiversity, inventory, hotspot, Jatinga, Assam.

\section{Introduction}

The census and conservation of biodiversity and its appropriate use are vital global issues. India is one of the twelve mega diversity countries having two hotspots viz., Eastern Himalaya and Western Ghats. The inventorization of the biota of these two hot spots is necessary for drawing various inferences, meant 
for conservation and habitat management. In this regard, the author has ventured to compile an inventory of certain Lepidopteran species referable to the subfamilies Pyraustinae, Catocalinae, Ophiderinae and Acronictinae from one locality -Jatinga ( $24^{\circ}$ to $28^{0} 18^{\prime} \mathrm{N}, 89^{\circ} 46^{\prime} \mathrm{E}$ to $\left.97^{\circ} 4^{\prime} \mathrm{E}\right)$ in northeastern India. Jatinga, a tiny village $(820 \mathrm{~m})$ is about $9 \mathrm{~km}$ away from Haflong in North Cachar Hills. It is connected by three roads, one from Lamatinga, the second from Silcher and the third from Haflong. Survey work was undertaken in the months of April, August and September mainly between 1982-1986 under the UGC and CSIR projects in some northeastern Indian localities. Upon scrutiny of these collections, Jatinga was found to be quite rich in moth diversity. The present inventory will be useful in devising suitable conservation and economic measures.

\section{Material and Methods}

The entire material during the studies were collected from the lights fitted at the bird watching tower at Jatinga. Sometimes a portable light trap or a petromax lamp (during failure of electricity) was also used. The adults were immediately killed after their collection with ethyl-acetate vapours in insect killing bottles of different sizes, depending upon the size of the specimen. In order to keep the scales intact on the body of the moths, they were removed from the bottles as soon as they were killed. Each of the killed specimens was pinned through the middle line of the thorax and the top of the pin was so adjusted in order to handle the specimens without the fingers touching it. This was proceeded by spreading of both the wings on the insect stretching board, followed by their drying in the improvised drying chambers fitted with electric bulbs or mild heaters. Each specimen was provided with a slip indicating the locality and date of collection. The specimens were preserved in airtight insect boxes, having naphthalene balls as fumigant. It may also be added that the setting of some specimens done in humid environment of Jatinga warranted their re-setting in the laboratory, as the wings tend to loose their original position. Such specimens were relaxed in the laboratory, reset and dried in an oven set at $40^{\circ} \mathrm{C}$. Various morphological characters such as head appendages, wing maculation, wing venation and genitalia were examined to identify different species.

\section{Observations}

Before the enlisting of different species is done, it seems appropriate to mention here that the works by Hampson (1896, 1899, 1908, 1912), Mehta (1933), Klima (1939), Munroe (1950, 1952, 1954, 1957, 1959, 1960), Nazmi (1963), Wang (1963), Pajni and Rose (1972, 1977, 1978), Bhattacherjee (1973, 1980) Rose and Pajni (1979), Rose (1982a, b), Rose and Dhillon (1980, 1982), Fletcher and Nye (1984), Rose and Singh (1985, 1989), Rose and Kirti (1986, 1987, 1989), Kirti and Rose (1987, 1989, 1990, 1992), Robinson et al. (1994), and Gupta (2000) have mainly been referred to for the identification of 81 species referable to 44 genera of the subfamily Pyraustinae. Thirty-nine species belonging to 24 genera of the subfamily Catocalinae have been identified from Hampson (1894, 1913a, b), Sevastopulo (1956), Shull and Nadkerny (1961), Kapur and Arora (1971), Nye (1975), Pajni et al. (1985), Holloway (1977), Edwards (1978), Banziger (1982) and, Rose and Kaur (1990). The identification of 44 species pertaining to 32 genera of the subfamily Ophiderinae (Butler, 1879, 1892; Moore, 1881; Hampson, 1894, 1912, 1924; Pierce, 1909, 1942; Wileman, 1922; Barnes \& Benjamin, 1926; Wileman \& West, 1928, 1929; Mehta, 1933; Ogata, 1954; Sugi, 1961; Nye, 1975; Bänziger, 1982, 1983, 1985; Pelletier, 1982; Kitching, 1984; Srivastava \& Rose, 1987, 1989, 1990, 1993a, b; Rose \& Srivastava, 1988, 1989a, b, 1994; Srivastava, 1992) and 16 species belonging to 12 genera of the subfamily Acronictinae (Moore, 1867, 1878; Butler, 1879; Swinhoe, 1889, 1895; Hampson, 1894, 1895, 1897, 1902, 1912, 1913a, b; Fletcher, 1957; Bayer, 1960; Chatterjee, 1967; Brown \& Dewhurst, 1975; Nye, 1975) has been affected by referring to the respective literature sources. As such, the list of 180 species compiled on the basis of collections housed in the Lepidoptera Laboratory in the Department of Zoology, Punjabi University, Patiala is as follows:

\section{Subfamily: Pyraustinae}

1. Aetholix flavibasalis (Guenée)

Guenée, 1854, in Boisduval \& Guenée, Hist. nat. Insectes (Spec. gén. Lépid.) 8: 193 (Male) (Aediodes) (Bombay).

Material examined: One female, 10.ix.1982; one female, 6.ix.1983. Wing expanse: Half: Female: $10 \mathrm{~mm}$.

\section{Agathodes ostentalis (Geyer)}

Geyer, 1837, in Hübner, Zuträge Samml. exot. Schmett. 5: 11, pl [143], figs.833, 834 (Perinephla) (Java).

Material examined: One male, one female, 21.v.1986-22.v.1986. Wing expanse: Half: Male: 15.50; Female: $16 \mathrm{~mm}$.

\section{Agrotera discinotata Swinhoe}

Swinhoe, 1894, Ann. Mag. nat. Hist. 14 (6): 207 (Male) (Agrotera) (Cheerapunjee).

Material examined: Two females, 14.ix.1985-16.ix.1985; 1female, 18.ix.1986.

Wing expanse: Half: Female: $11 \mathrm{~mm}$.

4. Arthroschista hilaris (Walker)

Walker, 1859, List Specimens lepid. Insects Colln Br. Mus. 18: 532 (Margaronia) (Ceylon).

Material examined: Four males, three females, 14.ix.198515.ix.1985.

Wing expanse: Half: Male: 28.50-29.50mm; Female: 24-27.50mm.

5. Bocchoris adipalis (Lederer)

Lederer, 1863, Wien. ent. Monatschr. 7: 376 (abipalis), 475, pl.11, fig.16 (Botys) (Amboina).

Material examined: One male, 12.ix, 1983.

Wing expanse: Half: Female: $11.50 \mathrm{~mm}$.

\section{Botyodes asialis (Guenée)}

Guenée, 1854, in Boisduval \& Guenée, Hist. nat. Insectis (Spec. 
gén. Lépid.), 8: 321 (Female) (Botyodes) (India).

Material examined: Two males, one female, 19.ix.1986.

Wing expanse: Half: Male: $44 \mathrm{~mm}$; Female: $40 \mathrm{~mm}$.

7. Chalcidoptera appensalis (Snellen)

Snellen, 1884, Tijdschr. Ent. 27: 41, pl.3, fig.12, 12a (Entephria)

(Celebes, Java).

Material examined: One male, 8.viii.1982: one male, two females,

3.ix.1983-5.ix.1983.

Wing expanse: Half: Male: $11 \mathrm{~mm}$; Female: $11 \mathrm{~mm}$.

8. Diaphania actorionalis (Walker)

Walker, 1859, List Specimens lepid. Insects Colln. Br. Mus. 17: 498 (Male) (Glyphodes) (India, Ceylon).

Material examined: Three males, 22.ix.1986.

Wing expanse: Half: Male: $14 \mathrm{~mm}$.

9. Diaphania canthusalis (Walker)

Walker, 1859, List Specimens lepid. Insects Colln. Br. Mus. 17: 505 (Male) (Glyphodes) (Sarawak).

Material examined: Two males, 15.ix.1985.

Wing expanse: Half: Male: $16 \mathrm{~mm}$.

10. Diaphania negatalis (Walker)

Walker, 1859, List Specimens lepid. Insects Colln. Br. Mus. 17: 458 (Female) (Phalangiodes ?) (Ceylon).

Material examined: Two males, 21.v.1986-26.v.1986.

Wing expanse: Half: Male: $12 \mathrm{~mm}$.

11. Diaphania zelleri (Lederer)

Lederer, 1863, Wien. ent. Monatschr. 7: 402, 478, pl.14, fig.3. (Male)

(Glyphodes) (Amboina).

Material examined: Two males, two females, 22.v.1986.

Wing expanse: Half: Male: $19 \mathrm{~mm}$; Female: $19 \mathrm{~mm}$.

12. Dichocrocis bistrigalis (Walker)

Walker, [1866] 1865, List Specimens lepid. Insects Colln. Br. Mus. 34: 1348 (Male) (Zebronia) (Darjeeling).

Material examined: Two males, one female, 17.v.1986-27.v.1986.

Wing expanse: Half: Male: $15 \mathrm{~mm}$; Female: $15 \mathrm{~mm}$.

13. Dichocrocis definita (Butler)

Butler, 1889, Illust. typical Specimens lepid. Heterocara Colln.

Br. Mus. 7: 97, pl.133, fig. 9 (Haritala).

Material examined: One male, one female, 20.v.1986-29.v.1986.

Wing expanse: Half: Male: $14.50 \mathrm{~mm}$; Female: $14.50 \mathrm{~mm}$.

14. Dichocrocis evaxalis (Walker)

Walker, 1859, List Specimens lepid. Insects Colln. Br. Mus. 19: 995 (Male) (Botys) (India).

Material examined: One male, two females, 21.v.1986-25.v.1986. Wing expanse: Half: Male: $12 \mathrm{~mm}$; Female: $14 \mathrm{~mm}$.

15. Dichocrocis nilusalis (Walker)

Walker, 1859, List Specimens lepid. Insects Colln. Br. Mus. 18: 695 (Male) (Botys) (Borneo).

Material examined: Three females, 8.ix.1983.

Wing expanse: Half: Female: $13-14 \mathrm{~mm}$.
16. Dichocrocis plutusalis (Walker)

Walker, 1859, List Specimens lepid. Insects Colln. Br. Mus. 17: 478 (Zebronia) (India).

Material examined: One male, 17.ix.1985; two males, 15.ix.1986. Wing expanse: Half: Male: $14 \mathrm{~mm}$.

17. Dichocrocis pyrrhalis (Walker)

Walker, 1859, List Specimens lepid. Insects Colln. Br. Mus. 17: 483 (Male) (Zebronia) (Sarawak).

Material examined: Two males, one female, 17.ix.1985.

Wing expanse: Half: Male: $13 \mathrm{~mm}$; Female: $13 \mathrm{~mm}$.

18. Dichocrocis rigidalis (Snellen)

Snellen, 1890, Trans. ent. Soc. Lond. 1890:631 (Female) (Zebronia) (Sikkim).

Material examined: One male, one female, 14.ix.1986-19.ix.1986.

Wing expanse: Half: Male: 13; Female: $13 \mathrm{~mm}$.

19. Dichocrocis semifascialis (Walker)

Walker, [1866] 1865, List Specimens lepid. Insects Colln. Br. Mus. 34: 1381 (Female) (Astura) (Moreton-Bai).

Material examined: Two males, 25.v.1986-27.v.1986; one male, three females, 15.ix.1986-21.ix.1986.

Wing expanse: Half: Male: $12 \mathrm{~mm}$; Female: $12 \mathrm{~mm}$.

20. Glyphodes caesalis Walker

Walker, 1859, List Specimens lepid. Insects Colln. Br. Mus. 17: 499 (Male) (Glyphodes) (Ceylon, India).

Material examined: Six males, two females, 3.ix.1983-12.ix.1983.

Wing expanse: Half: Male: $15 \mathrm{~mm}$; Female: $15 \mathrm{~mm}$.

21. Glyphodes multilinealis Kenrick

Kenrick, 1907, Proc. Zool. Soc. Lond. 1907:83, pl.4, fig.173 (Glyphodes).

Material examined: Three males, two females, 20.v.1985-21.v.1985; one male, one female, 15.ix.1986.

Wing expanse: Half: Male: mm: Female: $\mathrm{mm}$.

22. Glyphodes pulverulentalis Hampson

Hampson 1896, Fauna Br. India (Moths), 4: 353 (Male) (Glyphodes) (Nagas, Tenasserim).

Material examined: Six males, one female, 3.ix.1983-9.ix.1983.

Wing expanse: Half: Male: $14 \mathrm{~mm}$; Female: $14.50 \mathrm{~mm}$.

23. Glyphodes stolalis Guenée

Guenée, 1854, in Boisduval \& Guenée, Hist. nat. Insects (Spec. gén Lépid.) 8: 293, pl.3, fig.11 (Male) (Glyphodes) (India ?).

Material examined: Four males, three females, 4.ix.1983-7.ix-1983. Wing expanse: Half: Male: 17-19mm; Female: 17-19mm.

24. Hemopsis dissipatalis (Lederer)

Lederer, 1863, Wien. Ent. Monatschr. 7: 376, 474 (Female), pl.11, fig.13 (Botys) (Amboina).

Material examined: Three males, one female, 8.ix.1983-12.ix.1983. Wing expanse: Half: Male: $14 \mathrm{~mm}$; Female: $14 \mathrm{~mm}$.

25. Herpetogramma cynaralis (Walker)

Walker, 1859, List Specimens lepid. Insects Colln. Br. Mus. 18: 
672 (Botys).

Material examined: Three males, 13.ix.1986-17.ix.1986.

Wing expanse: Half: Male: $11 \mathrm{~mm}$.

26. Heterocnephes lymphatalis (Swinhoe)

Swinhoe, 1889, Proc. zool. Soc. Lond. 1889; 420, pl. 44, fig. 7

(Nosophora).

Material examined: One male, one female, 16.ix.1985.

Wing expanse: Half: Male: $14.50 \mathrm{~mm}$; Female: $14.50-15.50 \mathrm{~mm}$.

27. Hymenia perspectalis (Hübner)

Hübner, 1796, Samml. eur. Schmett. 6: 18, pl. 16, fig. 101 (Pyralis).

Material examined: Two males, two females, 25.v.1986-28.v.1986.

Wing expanse: Half: Male: 9mm; Female: $9 \mathrm{~mm}$.

28. Indogrammodes pectinicornalis (Guenée)

Guenée, 1854, in Boisduval \& Guenée, Hist. nat. Insectes (Spec. gén. Lépid.) 8:326 (Botys) (India).

Material examined: Three males, two females, 10.ix.1985-

13.ix.1985; 1?,??, 15.ix.1986.

Wing expanse: Half: Male: $14 \mathrm{~mm}$; Female $16 \mathrm{~mm}$.

29. Ischnurges angustalis Hampson

Hampson, 1893, Illust. typical Specimens lepid. Heterocera Colln Br. Mus. 9: 173, pl. 172, fig. 20 (Ischnurges).

Material examined: Two males, one female, 12.ix.1985-13.ix.1985;

1?,2??, 17.ix.1986.

Wing expanse: Half: Male: $10 \mathrm{~mm}$; Female: $10 \mathrm{~mm}$.

30. Ischnurges gratiosalils (Walker)

Walker, 1859, List Specimens lepid. Insects Colln. Br. Mus. 17: 357 (Male) (Samea) (Ceylon).

Material examined: One male, one female, 8.ix.1982.

Wing expanse: Half: Male: $11 \mathrm{~mm}$; Female: $12 \mathrm{~mm}$.

31. Lamprosema commixta (Butler)

Butler, 1879, Ann. Mag. nat. Hist. (5) 4: 453 (Samea) (Japan).

Material examined: One male, two females, 22.iv.1982-27.iv.1982.

Wing expanse: Half: Male: $8 \mathrm{~mm}$; Female: $9 \mathrm{~mm}$.

32. Lamprosema cuprealis (Moore)

Moore, 1877, Proc. zool. Soc. Lond. 1877: 616, pl. 60, fig. 13

(Female) (Coptobasis).

Material examined: Two males, two females, 8.ix.1982-10.ix.1982

Wing expanse: Half: Male: $13 \mathrm{~mm}$; Female: $13 \mathrm{~mm}$.

33. Limbobotys ptyophora (Hampson)

Hampson, 1896, Fauna Br. India (Moths) 4: 389, fig. 210 (Male)

(Crochidophora). (Sikkim, Tenasserim).

Material examined: Two males, one female, 12.ix.1985.

Wing expanse: Half: Male: $14 \mathrm{~mm}$; Female: $15 \mathrm{~mm}$.

34. Lygropia amplificata (Warren)

Warren, 1896, Ann. Mag. nat. Hist. 17 (6): 145 (Male) (Metoeca).

Material examined: Two males, one female, 26.v.1986; two males, two females, 12.ix.1986.

Wing expanse: Half: Male: $11 \mathrm{~mm}$; Female: $11 \mathrm{~mm}$.

35. Lygropia distorta (Moore)
Moore, 1885, Lepid. Ceylon 3:270, pl.150, fig. 7 (Pelecyntis) (Ceylon).

Material examined: Four males, 16.ix.1985.

Wing expanse: Half: Male: $18-25 \mathrm{~mm}$.

36. Maruca amboinalis (Felder \& Rogennofer)

Felder \& Rogenhofer, 1875, Reise öst. Fregatte Novara (Zool.) 2 (Abt.2): 18, pl. 135, fig. 24 (Male) (Siriocauta?) (Amboina).

Material examined: One female, 7.ix.1983.

Wing expanse: Half: Female: $15 \mathrm{~mm}$.

37. Meroctena tullalis (Walker)

Walker, 1859, List Specimens lepid. Insects Colln. Br. Mus. 18: 649 (Botys) (N. India).

Material examined: One male, two female, 13.ix.1985

Wing expanse: Half: Male: $19 \mathrm{~mm}$; Female: $20 \mathrm{~mm}$

38. Nagiella quadrimaculalis (Kollar)

Kollar, 1848, in C. F. von Hugel, Kaschmir und das Reich der Siek, Stuttgart 4 (2): 492 (Scopula).

Material examined: Two males, 29.v.1986.

Wing expanse: Half: Male: $14-17 \mathrm{~mm}$

39. Nausinoe perspectata (Fabricius)

Fabricius, 1775, Syst. Ent. 1775: 640 (Phalaena).

Material examined: One male, two females, 24.v.1986-25.v.1986; two males, 12.ix.1986-13.ix.1986.

Wing expanse: Half: Male: $13 \mathrm{~mm}$; Female: $12.50 \mathrm{~mm}$.

40. Nevrina procopia (Stoll)

Stoll, 1781, in Cramer, Uitlandsche Kapellen (Papillons exot.) 4: 152, pl.368, fig E (Phalaena).

Material examined: One male, two females, 22.v.1986.

Wing expanse: Half: Male: $19 \mathrm{~mm}$ : Female: $19 \mathrm{~mm}$.

41. Notarcha derogata (Fabricius)

Fabricius, 1775, Syst. Ent. 1775: 641 (Phalaena) (India).

Material examined: One male, two females, 21.ix.1985.

Wing expanse: Half: Male: $13 \mathrm{~mm}$; Female: $14 \mathrm{~mm}$.

42. Pagyda salvalis Walker

Walker, 1859, List specimens lepid. Insects Colln Br. Mus. 17: 487 (Pagyda) (Ceylon).

Material examined: One male, two females, 15.ix.1985-16.ix.1985. Wing expanse: Half: Male: $9 \mathrm{~mm}$; Female: $11 \mathrm{~mm}$.

43. Palpita pajnii Kirti \& Rose

Kirti \& Rose, 1992, J. Ent. Res. 16 (1): 72, figs 20, 21, 22 (Male gen.), 23 (Female gen.).

Material examined: One female, 10.ix.1983.

Wing expanse: Half: Female: $18 \mathrm{~mm}$

44. Palpita palpifulvata Kirti \& Rose

Kirti \& Rose, 1992, J. Ent. Res. 16 (1): 72, figs 8,9,10 (Male gen.), 11 (Female gen.).

Material examined: One female, 4.ix.1983.

Wing expanse: Half: Female: $11.50 \mathrm{~mm}$

45. Patania aurantiacalis (Fischer von Röslerstamm) 
Fischer von Röslerstamm, [1840] 1838, Abbildungeen Schmettkde (15): 213, pl. 75, fig.3 (Botys).

Material examined: Two males, two females, 23.v.1986-25.v.1986. Wing expanse: Half: Male: $14-15 \mathrm{~mm}$ : Female: $14-15 \mathrm{~mm}$.

46. Patania caletoralis (Walker)

Walker, 1859, List Specimens lepid. Insects Colln Br. Mus.18: 651 (Botys) (N. India).

Material examined: One male, one female, 26.v.1986.

Wing expanse: Half: Male: $17 \mathrm{~mm}$ : Female: $16.50 \mathrm{~mm}$

47. Patania concatenalis (Walker)

Walker, [1866] 1865, List Specimens lepid. Insects Colln Br. Mus. 34: 1408 (Female) (Botys) (Darjiling).

Material examined: Two males, two females, 21.v.1986-26.v.1986. Wing expanse: Half: Male: $13 \mathrm{~mm}$ : Female: $13 \mathrm{~mm}$.

48. Patania jatingaensis Rose \& Singh

Rose \& Singh, 1989, Entomon 14 (1\& 2): 39-43, figs. 6.

Material examined: Three males, 3.ix.1983-14.ix.1983; 1?, 23.ix.1983;2??, 13.ix.1985-15.ix.1985.

Wing expanse: Half: Male: $42 \mathrm{~mm}$.

49. Patania mysisalis (Walker)

Walker, 1859, List Specimens lepid. Insects Colln Br. Mus.18: 634 (Female) (Botys) (Sierra Leone).

Material examined: One male, three females, 25.v.1986-26.v.1986. Wing expanse: Half: Male: $13 \mathrm{~mm}$ : Female: $14 \mathrm{~mm}$.

50. Patania verecunda (Warren)

Warren, 1896, Ann. Mag. nat. Hist. 18 (6): 167, (Loxoscia) (Khasis).

Material examined: One male, two females, 25.v.1986-26.v.1986.

Wing expanse: Half: Male: $15 \mathrm{~mm}$ : Female: $14 \mathrm{~mm}$.

51. Phostria noctescens (Moore)

Moore, 1888, in Hewitson \& Moore, Descr. new Indian lepid.

Insects Colln late Mr. W.S. Atkinson (3): 218 (Charema)

(Darjiling).

Material examined: One female, 6.ix.1983.

Wing expanse: Half: Female: $18 \mathrm{~mm}$

52. Phostria unitalis (Guenée)

Guenée, 1854, in Boisduval \& Guenée, Hist. nat. Insectes (Spec. gén. Lépid.) 8: 349 (Male) (Botys) (India).

Material examined: Two males, 5.ix.1983.

Wing expanse: Half: Male: $11 \mathrm{~mm}$

53. Pionea ablactalis (Walker)

Walker, 1859, List Specimens lepid. Insects Colln Br. Mus. 18: 660 (Pionea).

Material examined: Two males, 22.v.1986.

Wing expanse: Half: Male: $14 \mathrm{~mm}$.

54. Pionea aureolalis (Lederer)

Lederer, 1863, Wien. ent. Monatschr. 7: 423 (Pionea).

Material examined: One female, 4.ix.1983.

Wing expanse: Half: Female: $11 \mathrm{~mm}$
55. Polythlipta euroalis (Swinhoe)

Swinhoe, 1889, Proc. zool. Soc. Lond. 1889: 420, pl. 44, fig. 12 (Nausinoe) (India).

Material examined: One male, 12.ix.1986.

Wing expanse: Half: Male: $16 \mathrm{~mm}$ : Female: $16 \mathrm{~mm}$.

56. Polythlipta inconspicua (Moore)

Moore, 1888, in Hewitson \& Moore, Descr. new Indian lepid. Insects Colln late Mr. Atkinson (3): 220 (Botyodes) (Darjiling). Material examined: One male, one female,16.ix.1985; two males, 12.ix.1986.

Wing expanse: Half: Male: 19mm: Female: $19 \mathrm{~mm}$.

57. Polythlipta macralis Lederer

Lederer, 1863, Wien. ent. Monatschr. 7: 389, 477, pl. 12. fig. 14 (Male) (Polythlipta) (Amboina).

Material examined: Two males, one female 15.ix.1985-16.ix.1985; one male, 12.ix.1986.

Wing expanse: Half: Male: $18 \mathrm{~mm}$ : Female: $18 \mathrm{~mm}$.

58. Polythlipta ossealis Lederer

Lederer, 1863, Wien. ent. Monatschr. 7: 389, pl. 12, fig. 18 (Polythlipta) (Amboina).

Material examined: Two males, 12.ix.1985; 2males, 15.ix.1986.

Wing expanse: Half: Male: $18 \mathrm{~mm}$.

59. Pramadea lunalis (Guenée).

Guenée, 1854, in Boisduval \& Guenée, Hist. nat. Insectes (Spec. gén. Lépid.) 8: 352 (Botys) (Coromandel).

Material examined: Two males, 8.iv.1982.

Wing expanse: Half: Male: $14 \mathrm{~mm}$.

60. Pramadea ovialis (Walker)

Walker, 1859, List Specimens lepid. Insects Colln Br. Mus. 18: 636 (Botys) (Congo).

Material examined: One male, 7.viii.1982.

Wing expanse: Half: Male: $13.50 \mathrm{~mm}$.

61. Prophantis octoguttalis (Felder \& Rogenhofer)

Felder \& Rogenhofer, 1875, Reise öst. Fregatte Novara (Zool.) 2 (Abt.2): pl. 135, fig. 38 (Male) (Botys) (Ambonia).

Material examined: Two males, 16.ix.1985; Two males, two females, 27.v.1986-29.v.1986; 1 male, 1 female, 12.ix.1986.

Wing expanse: Half: Male: $11 \mathrm{~mm}$ : Female: $11 \mathrm{~mm}$.

62. Prorodes mimica Swinhoe

Swinhoe, 1894, Ann. Mag. nat. Hist. (6) 14:205 (Prorodes)(India). Material examined: Four males, three females, 10.ix.1985-

13.ix.1985; 2 males, 2 females, 15.ix.1986.

Wing expanse: Half: Male: $17 \mathrm{~mm}$ : Female: $17 \mathrm{~mm}$.

63. Pycnarmon alboflavalis (Moore)

Moore, 1888, in Hewitson \& Moore, Descr. new Indian lepid. Insects Colln late Mr.W.S. Atkinson (3): 220 (Male) (Conogethes) (Darjiling).

Material examined: One male, 15.ix.1985.

Wing expanse: Half: Male: $10 \mathrm{~mm}$. 
64. Rhagoba octomaculalis (Moore)

Moore, 1867, Proc. zool. Soc. Lond. 1867: 95 (Female) (Filodes) (Darjiling).

Material examined: Four males, two females, 25.v.1986-26.v.1986; two males, one female, 14.ix.1986-16.ix.1986.

Wing expanse: Half: Male: 20mm: Female: 20-22mm

65. Samea castoralis (Walker)

Walker, 1859, List Specimens lepid. Insects Colln Br. Mus. 18: 693 (Female) (Botys) (Sarawak).

Material examined: One male, 21.v.1986; one male, one female, 10.ix.1986.

Wing expanse: Half: Male: $11 \mathrm{~mm}$ : Female: $11 \mathrm{~mm}$.

66. Satanastra aeriferalis (Moore)

Moore, 1877, Proc. zool. Soc. Lond. 1877: 618 (Conchylodes).

Material examined: One male, one female, 12.ix.1983.

Wing expanse: Half: Male: $11 \mathrm{~mm}$ : Female: $11 \mathrm{~mm}$.

67. Satanastra meritalis (Walker)

Walker, 1859, List Specimens lepid. Insects Colln Br. Mus. 17: 479 (Female) (Zebronia?) (Ceylon.).

Material examined: One female, 5.ix.1983.

Wing expanse: Half: Female: $9 \mathrm{~mm}$.

68. Satanastra virgitalis (Moore)

Moore, 1867, Proc. zool. Soc. Lond. 1867: 92, pl. 7, fig. 9 (Pycnarmon).

Material examined: Two males, 8.viii.1982; one male, 12.ix.1983.

Wing expanse: Half: Male: $10.50 \mathrm{~mm}$.

69. Sisyrophora pfeifferae Lederer

Lederer, 1863, Wien. ent. Monatschr. 7: 399, pl.3, fig.7; pl.5, fig.8; pl. 13, fig. 13 (Sisyrophora) (Singapore).

Material examined: One male, one female, 13.ix.1985-16.ix.1985.

Wing expanse: Half: Male: $18 \mathrm{~mm}$ : Female: $18 \mathrm{~mm}$.

70. Syllepte chromalis (Walker)

Walker, [1866]1865, List Specimens lepid. Insects Colln Br. Mus. 34: 1453 (Male) (Botys) (Java).

Material examined: Three females, 16.ix.1986.

Wing expanse: Half: Female: $15 \mathrm{~mm}$.

71. Syllepte iopasalis (Walker)

Walker, 1859, List Specimens lepid. Insects Colln Br. Mus. 18: 652 (Male) (Botys) (Burma).

Material examined: Four females, 12.ix.1986-16.ix.1986.

Wing expanse: Half: Female: $13 \mathrm{~mm}$.

72. Syllepte plagiatalis (Walker)

Walker, 1859, List Specimens lepid. Insects Colln Br. Mus. 18:673 (Botys).

Material examined: Two males, 10.ix.1985; two males, 15.ix.1986. Wing expanse: Half: Male: $13.50 \mathrm{~mm}$.

73. Syllepte sellalis (Guenée)

Guenée, 1854, in Boisduval \& Guenée, Hist. nat. Insectes (Spec. gén. Lépid.) 8: 330 (Female) (Botys) (Silhet).
Material examined: Five males, 16.ix.1985-20.ix.1985.

Wing expanse: Half: Male: $20 \mathrm{~mm}$.

74. Synclera traducalis (Zeller)

Zeller, 1852, Lepid. Microptera 54 (Eudioptis) (Limpop, Gariep). Material examined: One male, two females, 18.ix.1985.

Wing expanse: Half: Male: $19 \mathrm{~mm}$ : Female: $20 \mathrm{~mm}$.

75. Tangla eurytusalis (Walker)

Walker, 1859, List Specimens lepid. Insects Colln Br. Mus. 17: 503, (Female) (Gylphodes) (Sarawak).

Material examined: Three males, two females, 4.v.1986-26.v.1986; one male, one female, 13.ix.1986.

Wing expanse: Half: Male: $16 \mathrm{~mm}$ : Female: $16 \mathrm{~mm}$.

76. Tangla itysalis (Walker)

Walker, 1859, List Specimens lepid. Insects Colln Br. Mus. 17: 501, (Male) (Glyphodes) (Sarawak).

Material examined: Two males, two females, 8.ix.1985-14.ix.1985. Wing expanse: Half: Male: $15 \mathrm{~mm}$ : Female: $15 \mathrm{~mm}$.

77. Tangla naralis (Felder \& Rogenhofer)

Felder \& Rogenhofer, 1875, Reise öst. Fregatte Novara (Zool.) 2 (Abt.2): 10, pl. 136, fig. 38 (Glyphodes) (Sarawak).

Material examined: Two males, one female, 14.ix.1985-15.ix.1985. Wing expanse: Half: Male: $16 \mathrm{~mm}$ : Female: $16 \mathrm{~mm}$.

78. Tangla zangisalis (Walker)

Walker, 1859, List Specimens lepid. Insects Colln Br. Mus. 17: 504 (Male) (Glyphodes) (Sarawak).

Material examined: One male, 16.ix.1985; two males, 10.ix.198612.ix.1986.

Wing expanse: Half: Male: $14 \mathrm{~mm}$ : Female: $14 \mathrm{~mm}$.

79. Tangla zelimalis (Walker)

Walker, 1859, List Specimens lepid. Insects Colln Br. Mus. 17: 502, (Female) (Glyphodes) (Sarawak).

Material examined: Three males, two females, 15.ix.1985-16-ix1985; 1?, 2.ix.1986

Wing expanse: Half: Male: $15 \mathrm{~mm}$ : Female: $15 \mathrm{~mm}$.

80. Tetridia vinacealis (Moore)

Moore, 1877, Proc. zool. Soc. Lond. 1877: 619 (Botys)) (Port Blair).

Material examined: One male, one female, 4.ix.1983-6.ix.1983.

Wing expanse: Half: Male: $17 \mathrm{~mm}$ : Female: $16 \mathrm{~mm}$.

81. Toxobotys aureans Rose \& Kirti

Rose \& Kirti, 1989, J. Insect Sci. 2 (1): 10. figs.1-7.

Material examined: Two males, one female, 9.ix.1983.

Wing expanse: Half: Male: $15-16 \mathrm{~mm}$ : Female: $15 \mathrm{~mm}$.

\section{Subfamily: Catocalinae}

82. Achaea janata (Linnaeus)

Linnaeus, 1758, Syst. Nat. (Edn. 10) 1:527 (Phalaena).

Material examined: One male, one female, 14.v.1986- 21.v.1986; one male, three females, 11.ix.1986-16.ix.1986. 
Wing expanse: Half: Male: $31 \mathrm{~mm}$, Female: $31 \mathrm{~mm}$.

83. Achaea mercatoria (Fabricius)

Fabricius, 1775, Syst. Ent.: 604 (Noctua).

Material examined: Three males, 21.v.1986-28.v.1986; two females, 12.ix.1986.

Wing expanse: Half: Male: $33 \mathrm{~mm}$, Female: $31 \mathrm{~mm}$.

84. Alamis umbrina Guenée

Guenée, 1852, in Boisduval \& Guenée, Hist. nat. Insectes (Lépid.)

$7: 4$ (Alamis).

Material examined: One male, one female, 27.v.1986- 28.v.1986; one female, 14.ix.1986.

Wing expanse: Half: Male: 16-23 mm, Female: 17-29 mm.

85. Anisoneura hypocyanea Guenée

Guenée, 1852, in Boisduval \& Guenée, Hist. nat. Insectes (Lépid.)

7: 162 (Anisoneura).

Material examined: Five males, 15.ix.1985- 19.ix.1985; one male,

22.v.1985; three males, one female, 11.ix.1986- 16.ix.1986.

Wing expanse: Half: Male: $55 \mathrm{~mm}$, Female: $62 \mathrm{~mm}$.

86. Anиа coronata (Fabricius)

Fabricius, 1775, Syst. Ent.: 596 (Noctua).

Material examined: Four males, three females, 15.ix.1985; two males, three females, 21.v.1986; 18??, 5 ??, 10.ix.1986- 20.ix.1986. Wing expanse: Half: Male: 40-48mm, Female: $38-45 \mathrm{~mm}$.

\section{Artena dotata (Fabricius)}

Fabricius, 1794, Syst. Ent. 3(2) : 55 (Noctua).

Material examined: One female, 15.ix.1985; seven males, one female, 14.ix.1986-16.ix.1986.

Wing expanse: Half: Male: 40mm, Female: $39 \mathrm{~mm}$.

88. Caranilla onelia (Guenée)

Guenée, 1852, in Boisduval \& Guenée, Hist. nat. Insectes (Lépid.) 7:256 (Naxia).

Material examined: Six males, 25.v.1986- 28.v.1986; two males, two females, 11.ix.1986-12.ix.1986.

Wing expanse: Half: Male: $21 \mathrm{~mm}$, Female: $24 \mathrm{~mm}$.

89. Caranilla umbrosa (Walker)

Walker, 1865, List Specimens lepid. Insects Colln Br. Mus. 33: 968 (Ophiusa).

Material examined: One males, two females, 25.v.1986- 28.v.1986; two males, 10.ix.1986- 12.ix.1986.

Wing expanse: Half: Male: $21.50 \mathrm{~mm}$, Female: $22 \mathrm{~mm}$.

90. Chalciope mygdon (Cramer)

Cramer, 1777, Utilandsche Kapellen 2: 94, pl.156, fig.G., (Phalaena).

Material examined: One female, 3.ix.1985; 4 males, 13.ix.1985; six males, three females, 25.v.1986- 28.v.1986; one male, 12.ix.1986. Wing expanse: Half: Male: $20 \mathrm{~mm}$, Female: $17 \mathrm{~mm}$.

91. Cocytodes coerula Guenée

Guenée, 1852, in Boisduval \& Guenée, Hist. nat. Insectes (Lépid.) $7: 41$, pl.13, fig.10 (Cocytodes).
Material examined: One female, 27.v.1986; one female, 14.ix.1986. Wing expanse: Half: Female: $37-39 \mathrm{~mm}$.

92. Dysgonia algira (Linnaeus)

Linnaeus, 1767, Syst. Nat. (Edn 12) 1: 836 (Phalaena).

Material examined: Five males, 26.v.1986- 28.v.1986.

Wing expanse: Half: Male: $25 \mathrm{~mm}$.

93. Dysgonia properata (Walker)

Walker, 1858, List Speciemens lepid. Insects Colln Br. Mus. 14: 1433 (Ophiusa).

Material examined: One male, 13.ix.1986.

Wing expanse: Half: Male: $26 \mathrm{~mm}$.

94. Dysgonia sp.

Material examined: Three females, 25.ix.1986.

Wing expanse: Half: Female: $25-27 \mathrm{~mm}$.

95. Entomogramma torsa Guenée

Guenée, 1852, in Boisduval \& Guenée, Hist. nat. Insectes (Lépid.) 7: 204 (Entomogramma).

Material examined: Two males, 24.v.1986- 25.v.1986.

Wing expanse: Half: Male: $27 \mathrm{~mm}$.

96. Ercheia cyllaria (Cramer)

Cramer,1779, Uitlandsche Kapellan $\underline{3}: 100$, pl.251, fig.C, D (Noctua).

Material examined: Three males, two females, 13.ix.198519.ix.1985; 7??,4??, 10.ix.1986- 16.ix.1986.

Wing expanse: Half: Male: 24-26mm, Female: 27-29 mm.

97. Eupatula macrops (Linnaeus)

Linnaeus, 1768, Syst. nat. (Edn 12) 3: 225 (Phalaena).

Material examined: One male, 11.ix.1986.

Wing expanse: Half: Male: $73 \mathrm{~mm}$.

98. Grammodes geometrica (Fabricius)

Fabricius, 1775, Syst. Ent.: 599 (Noctua).

Material examined: Two males, two females, 23.v.1986- 28..1986.

Wing expanse: Half: Male: $24 \mathrm{~mm}$, Female: $21 \mathrm{~mm}$.

99. Hypopyra vespertilio (Fabricius)

Fabricius, 1787, Mantissa Insect. 2: 136 (Noctua).

Material examined: One male, 29.v.1986.

Wing expanse: Half: Male: $39 \mathrm{~mm}$.

100. Hypopyra sp.

Material examined: Two females, 21.v.1986.

Wing expanse: Half: Female: $39 \mathrm{~mm}$.

101. Lagoptera juno Dalman

Dalman, 1823, Analecta Ent. 52 (Noctua).

Material examined:One female, 15.ix.1985; one male, one female, 16.v.1986; two males, one female, 16.ix.1986.

Wing expanse: Half: Male: 45-47mm, Female: 50-54 mm.

102. Lygniodes ciliata Moore

Moore, 1867, Proc. zool. Soc. Lond. 1867: 69 (Lygniodes).

Material examined: One female, 17.ix.1985. 
Wing expanse: Half: Female: $46 \mathrm{~mm}$.

103. Lygniodes hypoleuca Guenée

Guenée, 1852, in Boisduval \& Guenée, Hist. nat. Insectes (Lépid.)

$7:$ 125, pl.16. fig. 5(Lygniodes).

Material examined: Ten males, four females, 17.ix.1985.

Wing expanse: Half: Male: $48 \mathrm{~mm}$, Female: $50 \mathrm{~mm}$.

104. Mocis frugalis (Fabricius)

Fabricius, 1775, Syst. Ent.: 601 (Noctua).

Material examined: One female, 17.ix.1985; two males, 22.v.1986-

27.v.1986; three females, 16.ix.1986.

Wing expanse: Half: Male: $20 \mathrm{~mm}$, Female: $19 \mathrm{~mm}$.

105. Mocis undata (Fabricius)

Fabricius, 1775, Syst. Ent.: 600 (Noctua).

Material examined: Three males, two females, 13.ix.1985-

19.ix.1985; three males, one female, 28.v.1986; twenty-two males,

10.ix.1986-16.ix.1986.

Wing expanse: Half: Male: $28 \mathrm{~mm}$, Female: $25 \mathrm{~mm}$.

106. Ophisma gravata Guenée

Guenée, 1852, in Bosiduval \& Guenée, Hist. nat. Insectes (Lépid.)

7: 237 (Ophisma).

Material examined: Five males, three females, 11.ix.1986-

19.ix.1986.

Wing expanse: Half: Male: $33 \mathrm{~mm}$, Female: $30 \mathrm{~mm}$.

107. Parallelia amygdalis (Moore)

Moore, 1887, Lepid. Ceylon 3 : 551, pl. 2214, fig.3 (Dysgonia).

Material examined: Three males, 19.v.1985-22.v.1985; two males,

27.v.1986- 28.v.1986.

Wing expanse: Half: Male: $23 \mathrm{~mm}$.

108. Parallelia analis (Guenée)

Guenée, 1852, in Boisduval \& Guenée, Hist. nat. Insectes (Lépid.)

$7: 271$ (Ophiusa).

Material examined: One male, 29.v.1986.

Wing expanse: Half: Male: $24 \mathrm{~mm}$.

109. Parallelia arcuata (Moore)

Moore, 1877, Proc. zool. Soc. Lond. 1877 : 609 (Ophiusa).

Material examined: One female, 10.ix.1985; 2 males, 24.v.1986-

28.v.1986; 2 ??, 1?, 14.ix.198-16.ix.1986.

Wing expanse: Half: Male: $26 \mathrm{~mm}$, Female: $25 \mathrm{~mm}$.

110. Parallelia crameri (Moore)

Moore, 1885, Lépid. Ceylon 3: 177, pl. 171, fig.2 (Dysgonia).

Material examined: One male, one female, 28.v.1986; 2 males,

11.ix.1986.

Wing expanse: Half: Male: $25 \mathrm{~mm}$, Female: $24 \mathrm{~mm}$.

111. Parallelia joviana (Stoll)

Stoll, 1782, Pap. Exot. IV: 273, p.399, fig. B. (Noctua).

Material examined: Two males,one female, 13.ix.1985- 15.ix.1985; female, 19.v.1986- 28.v.1986; four males, five females, 10.ix.198613.ix.1986.

Wing expanse: Half: Male: $25 \mathrm{~mm}$, Female: $27 \mathrm{~mm}$.
112. Parallelia maturescens (Walker)

Walker, 1858, List Specimens lepid. Insects Colln Br. Mus. 5 : 1382 (Ophisma).

Material examined: One male, one female, 28.v.1986; two males, 11.ix.1986.

Wing expanse: Half: Male: $25 \mathrm{~mm}$, Female: $24 \mathrm{~mm}$.

113. Parallelia sp. 1

Material examined: One male, 10.ix.1986.

Wing expanse: Half: Male: $25 \mathrm{~mm}$.

114. Parallelia sp. 2

Material examined: Two males, 28.v.1986.

Wing expanse: Half: Male: $22.50 \mathrm{~mm}$.

115. Parallelia $\mathrm{sp} .3$

Material examined: Two males, three females, 11.ix.1986-17.ix.1986.

Wing expanse: Half: Male: $31 \mathrm{~mm}$, Female: $32-33 \mathrm{~mm}$.

116. Pericyma sp.

Material examined: Eight males, three females, 13.ix.1985-

28.ix.1985; three males, two females, 20.v.1986-28.v.1986; fifteen males, eight females, 10.ix.1986-211.ix.1986.

Wing expanse: Half: Male: 21-22mm, Female: $21-24 \mathrm{~mm}$.

117. Phyllodes eyndhovi Vollerhoeven

Vollerhoeven, 1858, Tijdschr. Ent. 2: 86, pl. 61 (Phyllodes).

Material examined: One male, female, 29.v.1986.

Wing expanse: Half: Male: $61 \mathrm{~mm}$, Female: $64 \mathrm{~mm}$.

118. Pindara illibata (Fabricius)

Fabricius, 1775, Syst. Ent.: 592 (Noctua).

Material examined:One female, 13.ix.1985; one male, 21.v.1986; one male, five females, 10.ix.1986- 16.ix.1986.

Wing expanse: Half: Male: $36 \mathrm{~mm}$, Female: $34 \mathrm{~mm}$.

119. Thyas honesta Hübner

Hübner, [1824], Samml. exot. Schmett. 2: pl. [203], figs. 1, 2 (Thyas). Material examined: Eight males, one female, 21.v.1986- 27.v.1986; four males, 10.ix.1986- 16.ix.1986.

Wing expanse: Half: Male: $52 \mathrm{~mm}$, Female: $46 \mathrm{~mm}$.

120. Trigonodes hyppasia (Cramer)

Cramer, 1779, Uitlandsche Kapellan 3: 99, pl.250, fig E (Phalaena).

Material examined: Three females, 26.v.1986- 28.v.1986.

Wing expanse: Half: Female: $22 \mathrm{~mm}$

\section{Subfamily: Ophiderinae}

121. Aedia acronyctoides (Guenée)

Guenée, 1852, in Boisduval \& Guenée, Hist. nat. Insectes (Lépid.)

7: 47 (Catephia).

Material examined: Three males, 11.ix.1986- 14.ix.1986.

Wing expanse: Half: Male: $16 \mathrm{~mm}$.

122. Aedia discistriga (Walker)

Walker, [1858]1857, List. Specimens lepid. Insects Colln Br. Mus. 13: 1128 (Anophia). 
Material examined: Six males, 22.v.1986.

Wing expanse: Half: Male: $17 \mathrm{~mm}$.

123. Aedia lecucomelas (Linnaeus)

Linnaeus sensu Hübner [1803], Samml. eur. Schmett. 4: pl.62, fig. 304 (Noctua).

Material examined: Seven males, 21.v.1986-28.v.1986.

Wing expanse: Half: Male: $17 \mathrm{~mm}$.

124. Amphigonia hepatizans Guenée

Guenée, 1852, in Boisduval \& Guenée, Hist. nat. Insectes (Spec.

gén. Lépid.) 7: 338 (Amphigonia).

Material examined: Two males, 11.ix.1986-15.ix.1986.

Wing expanse: Half: Male: $29 \mathrm{~mm}$.

125. Anereuthina lilach Guenée

Guenée, 1852, in Boisduval \& Guenée, Hist. nat. Insects (Spec.

gén. Lépid.) 7: 260 (Anereuthina).

Material examined: One male, 12.ix.1985.

Wing expanse: Half: Male: $21 \mathrm{~mm}$.

126. Anomis banzigeri Srivastava \& Rose

Srivastava \& Rose, 1989, Entomon 14(3 \& 4): 238, figs. 4-7.

Material examined: One male, 17.ix.1985.

Wing expanse: Half: Male: $19 \mathrm{~mm}$.

127. Anomis fulvida Guenée

Guenée, 1852,in Boisduval \& Guenée, Hist. nat. Insectes (Spec. gén. Lépid.) 6: 397 (Anomis).

Material examined: One male, 17.ix.1985; one female, 11.ix.1986.

Wing expanse: Half: Male: $23 \mathrm{~mm}$, Female $21 \mathrm{~mm}$.

128. Anomis lineosa (Walker)

Walker, 1865, List Specimens lepid. Insects Colln Br. Mus. 33:

862 (Cosmophila).

Material examined: Two males, 17.ix.1985; two females, 25.v.1986.

Wing expanse: Half: Male: $23 \mathrm{~mm}$, Female 25mm.

129. Asta quadrilinea Walker

Walker, [1863] 1864, J. Proc. Linn. Soc. (Zool.) 7:170 (Asta).

Material examined: Four males, 31.viii.1986- 11.ix.1986.

Wing expanse: Half: Male: $24 \mathrm{~mm}$.

130. Bamra albicola (Walker)

Walker, 1858, List Specimens lepid. Insects Colln Br. Mus. 14 :

1010 (Felinia).

Material examined: Four males, 21.v.1986- 25.v.1986.

Wing expanse: Half: Male: $25 \mathrm{~mm}$.

131. Bamra sp.

Material examined: Three males, 21.v.1986.

Wing expanse: Half: Male: $27 \mathrm{~mm}$.

132. Calyptra minuticornis (Guenée)

Guenée, 1852, in Boisduval \& Guenée, Hist. nat. Insectes (Spec. gén. Lépid.) $6: 674$ (Calpe).

Material examined: One male, 19.ix.1986.

Wing expanse: Half: Male: $24 \mathrm{~mm}$.
133. Chrysopera combinans (Walker)

Walker, 1858, List Specimens lepid. Insects Colln Br. Mus. 14:1399 (Achaea).

Material examined: Six males, 15.ix.1985; one female, 10.ix.1986. Wing expanse: Half: Male: $24 \mathrm{~mm}$, Female $25 \mathrm{~mm}$.

134. Delgamma ampullonia Srivastava \& Rose

Srivastava \& Rose, 1990, Ad. Bios. 1990: 85,86, figs. 1-5.

Material examined: Fourteen males, three females, 13.ix.198516.ix.1985.

Wing expanse: Half: Male: $23 \mathrm{~mm}$, Female $25 \mathrm{~mm}$.

135. Delgamma pangonia Guenée

Guenée, 1852, in Boisduval \& Guenée, Hist. nat. Insectes (Spec. gén. Lépid.) 7:214 (Delgamma).

Material examined: Twelve males, six females, 13.ix.1985-

16.ix.1985.

Wing expanse: Half: Male: $25 \mathrm{~mm}$, Female $26 \mathrm{~mm}$.

136. Episparina tortuosalis (Moore)

Moore, 1867, Proc. zool. Soc. Lond. 1867: 81, pl.7, fig.5.

Material examined: Three males, seven females, 21.v.1986-

29.ix.1986.

Wing expanse: Half: Male: $28 \mathrm{~mm}$, Female $30 \mathrm{~mm}$.

137. Episparis exprimens Walker

Walker, 1862, Trans. ent. Soc. Lond. (3)1: 106.

Material examined: One male, 29.v.1986.

Wing expanse: Half: Male: $25 \mathrm{~mm}$.

138. Episparis liturata (Fabricius)

Fabricius, 1787, Mantissa Insect. 2: 197 (Phalaena).]

Material examined: Two males, three females, 28.v.1986.

Wing expanse: Half: Male: $23 \mathrm{~mm}$, Female $26 \mathrm{~mm}$.

139. Ericeia inangulata (Guenée)

Guenée, 1852, in Boisduval \& Guenée, Hist. nat. Insectes (Spec.gén. Lépid.) 7: 210 (Hulodes).

Material examined: Two females, 27.v.1986.

Wing expanse: Half: Female $27 \mathrm{~mm}$.

140. Eudocima salaminia (Cramer)

Cramer, 1777, Uitlandsche Kapellen 2:177, pl.174, fig.A (Phalaena).

Material examined: Six males, 15.ix.1985-16.ix.1985.

Wing expanse: Half: Male: $47 \mathrm{~mm}$.

141. Fodina stola Guenée

Guenée, 1852, in Boisduval \& Guenée, Hist. nat. Insectes (Spec. gén. Lépid.) 7: 275 (Fodina).

Material examined: One female, 24.v.1986.

Wing expanse: Half: Female 23mm.

142. Hamodes aurantiaca (Guenée)

Guenée, (1852), in Boisduval \& Guenée, Hist. nat. Insectes (Spec. gén. Lépid.) 7:203 (Hamodes).

Material examined: Three males, one female, 12.ix.1986- 14.ix.1986. Wing expanse: Half: Male: $33 \mathrm{~mm}$, Female $34 \mathrm{~mm}$. 


\section{Hulodes caranea (Cramer)}

Cramer, 1780, Uitlandsche Kapellen 3:140, 174, pl.269, figs. E, F (Phalaena).

Material examined: Two males, 17.ix.1985; three females, 10.ix.1986.

Wing expanse: Half: Male: $42 \mathrm{~mm}$, Female $40 \mathrm{~mm}$.

144. Hulodes sp.

Material examined: One female, 13.ix.1985.

Wing expanse: Half: Female $34 \mathrm{~mm}$.

145. Hypocala deflorata (Fabricius)

Fabricius, 1794, Ent. Syst. 3 (2): 127 (Hyblaea).

Material examined: Two males, 27.v.1986.

Wing expanse: Half: Male: $19 \mathrm{~mm}$.

\section{Hypocala moorei Butler}

Butler, 1892, Ann. Mag. nat. Hist. 6: 21 (Hypocala).

Material examined: Eleven males, 21.v.1986; seven females, 15.ix.1986.

Wing expanse: Half: Male: $25 \mathrm{~mm}$, Female $24 \mathrm{~mm}$.

147. Hypocala subsatura Guenée

Guenée, 1852, in BoisduvaL \& Guenée, Hist. nat. Insectes (Spec. gén.Lépid.) 7: 75 (Hypocala).

Material examined: Twenty males, seven females, 13.ix.198516.ix.1985.

Wing expanse: Half: Male: $22 \mathrm{~mm}$, Female $23 \mathrm{~mm}$.

148. Hypocala violacea Butler

Butler, 1879, Trans. ent. Soc. Lond. 1879: 6 (Hypocala).

Material examined: Forty-six males, fourteen males, 13.ix.1985-

16.ix.1985.

Wing expanse: Half: Male: 24mm, Female 23mm.

149. Hypospila bolinoides Guenée

Guenée, 1852, in Boisduval \& Guenée, Hist. nat. Insectes (Spec. gén. Lépid.) 7:358 (Hypospila).

Material examined: One male, 16.ix.1986.

Wing expanse: Half: Male: $21 \mathrm{~mm}$.

150. Ischyja manlia (Cramer)

Cramer, 1776, Uitlandsche Kapellen 1: 144, pl.92, fig.A

(Phalaena).

Material examined: Three males, 15.ix.1985; two females, 12.ix.1986.

Wing expanse: Half: Male: $50 \mathrm{~mm}$, Female $48 \mathrm{~mm}$.

151. Koptoplax lindsayi Hampson

Hampson, 1891, Illust. typical specimens Lepid. Heterocera Colln Br. Mus. 8: 33, 127, pl.154, fig.17 (Koptoplax).

Material examined: One male, 12.ix.1986.

Wing expanse: Half: Male: $24 \mathrm{~mm}$.

152. Lacera alope (Cramer)

Cramer, 1780, Uitlandsche Kapellen 3: 168, pl.286, figs. E,F

(Phalaena).

Material examined: One male, one female, 11.ix.1986- 17.ix.1986.
Wing expanse: Half: Male: $28 \mathrm{~mm}$, Female $27 \mathrm{~mm}$.

153. Ommatophora luminosa (Cramer)

Cramer, 1780, Uitlandsche Kapellen 3: 147, pl.274, fig. D

(Phalaena).

Material examined: Two males, 15.ix.1985; two males, 15.ix.1986.

Wing expanse: Half: Male: $31 \mathrm{~mm}$.

154. Oraesia emarginata (Fabricius)

Fabricius, 1794, Ent. Syst. 3 (2): 82 (Noctua).

Material examined: Three males, 20.v.1986; two females, 11.ix.1986.

Wing expanse: Half: Male: $22 \mathrm{~mm}$, Female $21 \mathrm{~mm}$.

155. Othreis cajeta (Cramer)

Cramer, 1779, Pap. Exot. 1: 48, pl.30, figs. A,B,O, C, ?(Phalaena). Material examined: One male, one female, 16.ix.1986- 17.ix.1986. Wing expanse: Half: Male: $47 \mathrm{~mm}$, Female $45 \mathrm{~mm}$.

156. Othreis fullonia (Clerck)

Clerck, 1764, Icones Insect. rariorum 2: 40 (Phalaena).

Material examined: Fourteen males, three females, 10.ix.1986-

16.ix.1986,

Wing expanse: Half: Male: $48 \mathrm{~mm}$, Female $51 \mathrm{~mm}$.

157. Oxyodes scrobiculata (Fabricius)

Fabricius, 1775, Syst. Ent. 1775: 592 (Noctua).

Material examined:Twelve males, 10.v.1986; two females, 10.ix.1986.

Wing expanse: Half: Male: 30mm, Female 29mm.

158. Platyja umminia (Cramer)

Cramer, 1780, Uitlandsche Kapellen 3: 137, pl.267, fig. F. (Phalaena).

Material examined: Two males, 15.ix.1985; three females, 16.ix.1986.

Wing expanse: Half: Male: $30 \mathrm{~mm}$, Female $33 \mathrm{~mm}$.

159. Ramadasa pavo (Walker)

Walker, 1856, List Specimens lepid. Insects Colln Br. Mus. 9: 147 (Chasmina).

Material examined: Seven males, three females, 10.ix.198621.ix.1986.

Wing expanse: Half: Male: $24 \mathrm{~mm}$, Female $25 \mathrm{~mm}$.

160. Rhytia hypermnestra (Stoll)

Stoll, 1780, Papillons exot. 4:69, index pl. 233, figs. A,B

(Phalaena).

Material examined: Six males, 15.ix.1985.

Wing expanse: Half: Male: $44 \mathrm{~mm}$.

161. Serrodes campana Guenée

Guenée, 1852, in Boisduval \& Guenée, Hist. nat. Insectes (Spec.gén.Lépid.) 7: 252 (Serrodes).

Material examined: Two males, 16.ix.1986.

Wing expanse: Half: Male: $34 \mathrm{~mm}$.

162. Speiredonia alix Guenée

Guenée, 1852, in Boisduval \& Guenée, Hist. nat. Insectes 
(Spec.gén.Lépid.) 7: 171 (Speiredonia).

Material examined: Four males, 24.v.1986- 28.v.1986.

Wing expanse: Half: Male: $31 \mathrm{~mm}$.

163. Sympis rufibaris Guenée

Guenée, 1852, in Boisduval \& Guenée, Hist. nat. Insectes (Spec.gén Lépid.) 7: 343 (Sympis).

Material examined: Two males, 13.ix.1985; 11??, 27.v.1986.

Wing expanse: Half: Male: 22mm, Female 23mm.

164. Trichopolydesma sp.Material examined: 3??, 21.v 1986.

Wing expanse: Half: Male: $23 \mathrm{~mm}$.

\section{Subfamily: Acronictinae}

165. Acronicta maxima (Moore)

Moore, 1881, Proc. zool. Soc. Lond. 1881: 333 (Triaena).

Material examined: Five males, two females, 21.v.1986- 27.v.1986; two males, 11.ix.1986.

Wing expanse: Half: Male: $35 \mathrm{~mm}$, Female: $37 \mathrm{~mm}$.

166. Apsarasa radians (Westwood)

Westwood, 1848, Cabinet Oriental Ent.: 58, pl. 28, Fig. 4 (Noctua).

Material examined: Five males, 22.v.1986- 28.v.1986; four males, 10.ix.1986-12.ix.1986.

Wing expanse: Half: Male: $24 \mathrm{~mm}$.

167. Athetis cognata (Moore)

Moore, 1882, in Hewitson \& Moore, Descr. new Indian lepid. Insects Colln late Mr. W.S. Atkinson (2): 119 (Graphiophora). Material examined: Three males, 20.v.1986- 27.v.1986.

Wing expanse: Half: Male: $12 \mathrm{~mm}$.

168. Athetis reclusa (Walker)

Walker, 1862, J. Proc. Linn. Soc. (Zool. ) 6: 185 (Prodenia).

Material examined: Two males, 22.v.1986.

Wing expanse: Half: Male: $18 \mathrm{~mm}$.

169. Callopistria exotica (Guenée)

Guenée, 1852, in Boisduval \& Guenée, Hist. nat. Insectes (Spec. gén. Lépid), 7: 294 (Eriopus).

Material examined: Four males, two females, 11.ix.1986- 12.ix.1986. Wing expanse: Half: Male: $16 \mathrm{~mm}$, Female: $15 \mathrm{~mm}$.

170. Callopistria recurvata Moore

Moore, 1882, in Hewitson \& Moore, Descr. new. Indian. lepid. Insects Colln. Late Mr. W.S. Atkinson (2): 44 (Callopistria).

Material examined: Five males, two females, 27.v.2986; 8??, 10.ix.1986- 12.ix.1986.

Wing expanse: Half: Male: $17 \mathrm{~mm}$, Female: $15 \mathrm{~mm}$.

171. Callyna monolecuca Walker

Walker, 1858, List Specimens lepid. Insects Colln. Br. Mus. 15 : 1667 (Callyna).

Material examined: Three males, two females, 21.v..198625.v.1986; one male, one female, 11.ix.1986.

Wing expanse: Half: Male: $21 \mathrm{~mm}$, Female: $23 \mathrm{~mm}$.
172. Chasmina tibialis (Fabricius)

Fabricius, 1775, Syst. Ent.: 578 (Bombyx).

Material examined: Three males, 21.v.1986- 22.v.2986; two males, three females, 11.ix.1986- 12.ix 1986.

Wing expanse: Half: Male: $25 \mathrm{~mm}$, Female: $24 \mathrm{~mm}$.

173. Craniophora fasciata Moore

Moore, [1885] 1887, Lepid. Ceylon 3: 5, pl.144, Fig. 4 (Acronycta). Material examined: Seven males, 10.ix.1986- 16.ix.1986.

Wing expanse: Half: Male: $20 \mathrm{~mm}$.

174. Dipterygina indica (Moore)

Moore, 1867, Proc. zool. Soc. Lond. 1867 : 78, pl. 51 (Dipterygia). Material examined: One male, 27.v.1986; two males, 14.ix.198619.ix.1986.

Wing expanse: Half: Male: $20 \mathrm{~mm}$.

175. Eutamsia indistans (Guenée)

Guenée, 1852, in Boisduval \& Guenée, Hist. nat. Insectes (Spec. gén. Lépid.) 6: 87 (Hadena).

Material examined: One female, 26.v.1986; one male, 16.ix.1986.

Wing expanse: Half: Male: $18 \mathrm{~mm}$, Female: $20 \mathrm{~mm}$.

176. Prospalta sp.

Material examined: One male, 26.v.1986; three males, 26.ix.198628.ix.1986.

Wing expanse: Half: Male: 14-17mm.

177. Spodoptera littoratis (Boisduval)

Boisduval, 1853, Nouv. Annls Mus. Hist. nat. Paris 2: 239, pl. 13.

Fig.8. (Hadena).

Material examined:Three males, one female, 14.ix.1986-16.ix.1986. Wing expanse: Half: Male: $22-23 \mathrm{~mm}$, Female: $21 \mathrm{~mm}$.

178. Spodoptera pecten Guenée Guenée, 1852, in Boisduval \& Guenée, Hist nat. Insectes (Spec. gén. Lépid.), 7:155 (Spodoptera).

Material examined: Two females, 27.v.1986.

Wing expanse: Half: Female: $16 \mathrm{~mm}$.

179. Spodoptera quadripunctata (Fabricius)

Fabricius, 1775, Syst. Ent. : 594 (Caradrina).

Material examined: Two males, 21.v.1986- 27.v.1986.

Wing expanse: Half: Male: $14.50 \mathrm{~mm}$.

180. Yepcalphis dilectissima (Walker)

Walker, 1858, List Specimens lepid. Insects Colln Br. Mus. 15: 1751 (Ariola).

Material examined: One female, 25.v.1986.

Wing expanse: Half: Female: $13 \mathrm{~mm}$.

\section{Remarks}

Out of 180 species listed above, the species Dichocrocis bistrigalis, D. pyrrhalis, Heterogramma cynaralis, Lygropia distorta, Pagyda salvalis, Pionea ablactalis, Pycnarmon alboflavalis, Syllepte chromalis (Pyraustinae); Achaea mercatoria, Dysgonia algira, Parallelia analis (Catocalinae); Amphigonia hepatizana, Chrysopera combinans, Delgamma 
pangoina, Hypocala deflorata (Ophiderinae) and Athetis reclusa, Craniophora fasciata, Dipterygina indica, Yepcalphis dilectissima (Acronictinae) of the respective subfamilies are new records from northeastern India. Further, besides furnishing additional collection data for the species Dichocrocis definita, Heterocnephes lymphatalis, Indogrammodes pectinicornalis, Limbobotys ptyophora, Meroctena tuttalis, Nagiella quadrimaculalis, Nevrina procopia, Patania caletoralis, $P$. concatenalis, $P$. mysisalis, Polythlipta euroalis, $P$. inconspicua, P. ossealis, Prophantis octoguttalis, Samea castoralis, Tangla naralis, T. zangisalis, T. zelimalis, the species Polythlipta macralis, Tangla itysalis, Diaphania canthusalis, D. zelleri, Sisrophora pfeifferae, Dichocrocis rigidalis, Hymenia perspectalis, Patania verecunda, P. auranticalis, Notarcha derogata and Prorodes mimica are recorded from Jatinga for the first time (Kirti \& Sodhi, 2001). Besides, the species at serial numbers 125, 129 (Ophiderinae) and 169 (Acronictinae) are new records from India. Similarly the Pyraustin species mentioned at Sr. nos. 1, 2, 4, 6, 23, 24, 27, 28, 40, 42, 47, 57, 61, 62, 64, 69, 74, 78, and 80 (Fletcher \& Nye, 1984); those of Catocalinae listed at serial 84, 88, 90, 91, 92, 97, 98, 99, 106, 118, 119, 120; those of Ophiderinae at 123, 124, 129, 133, 140, 143, 145, 149, 150, 151, 152, 153, 154, 157, 158, 159; and those of Acronictinae at 166 and 175 (Nye, 1975) which are the type species of an equal number of valid genera are also available in this very locality. However, it may be clarified that Jatinga be cautiously taken as a topotype locality for these species.

Further, like other species, though all the Ophiderin species have been collected from light yet it seems appropriate to add for information sake that the species such as Eudocima salaminia (papaya, guava), Ischyja manlia (guava), Oraesia emarginata (guava), Othreis fullonia (mainly citrus, also guava and peach), Platyja umminia (guava, peach, rose apple) and Rhytia hypermnestra (mainly grapes, also guava) infest and damage the respective fruits, mentioned in parentheses. Species such as Anomis lineosa, Ericeia inangulata, Othreis cajeta and Oxyodes scrobiculata are polyphagous, as they suck the juices from a variety of fruit sources (Hargreaves, 1936; Ayyar, 1944; Pruthi, 1969; Atwal, 1986; Nayar et al., 1983). Further, Bänziger (1979) while reporting Calyptra minuticornis near tropical monsoon forests in the hilly regions of northern Thailand and tropical evergreen and semi-evergreen rain forests of southern Thailand and northwestern Malysia noticed this species attacking the elephant, water buffalo, zebu and tapir. The species has also been suspected to be fruit piercing moth in Indonesia (Kalshoven, 1951) and lachryphagous in Cambodia (Büttiker, 1962). Interestingly and dangerously, the same species are being reported from Jatinga as well.

On the basis of present inventory comprising 180 species referable to 112 genera from such a small locality should not only evoke interest in taxonomic and behavioral studies on the adults but also on other biological and life history aspects. The role of non-pest species in the conservation of a habitat need hardly be emphasized. The larval hosts of such species need to be located, as we do not have much knowledge about the immature stages of moths in India. The information on flowers being visited by different species for nectar sucking and incidental pollination is another gap area that needs to be plugged.

As such, Jatinga in North Cachar Hills (Assam) is an area rich in insect biodiveristy and due attention need to be given to this particular habitat while framing/ handling National Biodiversity Strategy and Action Plan (Khoshoo, 1995; Gadgil, 1996; MoEF, 1998; Kothari, 2001). It is being suggested through this communication that Jatinga be taken as a serious hotspeck in northeastern India.

Should it also be mentioned that Jatinga is erroneously known as place where birds commit suicide? It presents unique foggy weather conditions during the month of September. Besides having an experience of bird watching during these foggy weather conditions, the author has seen enormous insect diversity in different stages of their development. The months of May, October and September are comparatively better for making insect collections for scientific purposes. It may also be added that the local people exploit and hunt the beautiful bird diversity during these foggy environmental conditions at night. It is a pathetic scene to watch the birds being killed by them. In fact, during these confusing weather conditions, the birds leave their nests and strike against electric lights at night. Perhaps on the basis of this very notion, the observation stands that the birds come to this locality for committing suicide. The local people also erect artificial light sources such as petromax lamps to obstruct the flying birds to exploit these environmental conditions. To the best of my knowledge, the phenomenon though occurs at a few other places as well, needs more studies/ observations, particularly the scientific one. Besides entomologists, Jatinga is a spot, which warrants attention of ornithologists as well.

\section{Acknowledgements}

The author is grateful to the University Grants Commission, New Delhi for funding the project on Pyraustinae besides sanctioning a special museum grant to identify this fauna from NHM, London (formerly BMNH). Similarly, the financial assistance provided by CSIR, New Delhi is acknowledged gratefully for sanctioning the project on noctuid moths. The cooperation extended by the authorities of Zoological Survey of India, Calcutta; Forest Research Institute, Dehradun and Indian Agricultural Research Institute, New Delhi is acknowledged gratefully. The much-needed assistance offered by the then staff of the two above mentioned projects also deserves appreciation for undertaking surveys. Thanks are due 
to my teacher, Professor (Retd.) H.R. Pajni, Department of Zoology, Punjab University, Chandigarh, who introduced me to the fascinating world of moths and also supported my idea of publishing this inventory and above all, going though the final draft of this manuscript. Thanks are due to Dr. J.D. Holloway (London) for his comments on the identification of certain noctuid species and Dr. Bänziger (Thailand) for sending reprints. The recognition of the Lepidoptera Laboratory as a Coordinating Centre for undertaking research on Microlepidoptera under the All India Coordinating Project on Taxonomy (AICOPTAX) and funding of the project by the Ministry of Environment \& Forests, New Delhi is acknowledged gratefully. For typing and setting the matter, the author would like to thank his son, Mr. Onkarpreet Singh .

\section{References}

Atwal, A.S. (1986). Agricultural Pests of India and South-East Asia. $2^{\text {nd }}$ edition. Kalyani Publishers, Ludhiana, 508 pp.

Ayyar, R. (1944). Notes on some fruit sucking moths of Deccan. Indian Journal of Entomology 5(1\&2): 29-33.

Bänziger, H. (1979). Skin-piercing blood- sucking moths II: Studies on further 3 adult Calyptera [Calpe]spp. (Lepid., Noctidae). Acta Tropica 36: 23-37.

Banziger, H. (1982). Fruit-piercing moths (Lepidoptera: Noctuidae) in Thailand: A general survey Andsomenew perspectives. Mitt. Schweiz. Ent. Ges. 55: 213-240.

Bänziger, H. (1983). A taxonomic revision of the fruit-piercing and blood-sucking moth genus Calyptera (=Calpe) (Lep., Noctudae). Entomon Scandinavia 14(4): 467-491.

Bänziger, H. (1985). Description of Otheris srivijayana, new species and notes on related fruit-piercing moths of Indo-Malayan region (Lep., Noctuidae). Heterocera Sumatrana, 2(0): 41-48. and some new perspectives. Mitt. Schweiz. Ent. Ges. 55: 213-240.

Barnes, W. and F.H. Benjamin (1926). On the genus Anomis (Lepidoptera, Phalaenidae). Entomology News Philadelphia 37: 78-81. Bayer, M.B (1960). The valves of the male genitalia in genera Prodenia, Laphygma and Spodoptera (Lepidoptera: Noctuidae). South African Journal of Agricultural Science 3: 625-631.

Bhattacharjee, N.S. (1973). A new species of genus Hymenia Hübner (lepidoptera: Pyralidae) from India. Indian Journal of Entomology 35 (1): 47-49.

Bhattacharjee, N.S. (1980). Studies on Indian Pyralidae 2. Identity and nomenclature of the family group Pyralidae and subfamily Pyraustinae. Bulletin of Entomological Society of India 21(1-2): 135137.

Brown, E.S.and C.F. Dewhurst (1975). The genus Spodoptera (Lepidoptera: Noctuidae) in Africa and the near east. Bulletin of Entomology Research 65(2): 221-262, 9 figs., 3 pls., 4 tabs.

Butler, A.G. (1879). On the collection of Lepidoptera from Cachar, North East India. Transactions of the Entomological Society of London 1879: 1-8.

Butler, A.G. (1892). On the genus Hypocala, a group of Noctuid moths. Annual Magazine of Natural History 6(10): 17-22.

Büttiker, W. (1962). Biological and morphological notes on the fruitpiercing and eye-frequenting moths. Verh. Int. Kongr. Ent. Wien. 1960 2: $10-15$.
Chatterjee,S.N. (1967). The identity of Spodoptera mauritia acronyctoides Guenée, Spodoptera pecten Guenée and Spodoptera abyssinia Guenée (Lepidoptera:Noctuidae) based Edwards, E.d. (1978). A review of the genus Achaea Hübner in Australia (Lepidoptera: Noctuidae). Journal of Australian Entomological Society 17(4): 329340.

Fletcher, D.S. (1957). Spodoptera mauritia (Boisduval) and Spodoptera triturata (Walker): two distinct species. Bulletin of Entomology Research 47:215-217.

Fletcher, D.S. (1957). On the study of male and female genitalia Proceedings of National Institute of Science, India (Biology) 35(1):3552.

Fletcher, D.S. and I.W.B. Nye (1984). The Generic Names of Moths of the World, 5: xv: $185 \mathrm{pp}$. (Trustees of the British Museum), London. Gadgil, M. (1996). Documenting diversity: An experiment. Current Science 70(1): 36-44.

Gupta, S.L. (2000). Pyraustinae (Lepidoptera: Pyralidae) fauna of Andaman and Nicobar Islands (India). Uttar Pradesh Journal of Zoology 20 (3): 269-273.

Hampson, G.F. (1894). Fauna of British India (Moths) 2: 609 pp. Taylor and Francis, London.

Hampson, G.F. (1895). Description of new Heterocera from India. Transactions of the Entomology Society of London 1895: 277-315.

Hampson, G.F. (1896). Fauna of British India (Moths) 4: xxviii + 594pp, figs. 287. Taylor and Francis, London.

Hampson, G.F. (1897). The moths of India. Supplementary paper to the volumes in the "Fauna of British India" Part II. Journal of the Bombay Natural History Society 11: 438-462.

Hampson, G.F. (1899). A revision of the subfamily Pyraustinae of family Pyralidae. Proceedings of the Zoological Society of London pp. 172-268.

Hampson, G.F. (1902). The moths of India. Supplementary paper to the volumes in the Fauna of British India Series II, Part V. Journal of the Bombay Natural History Society 14: 103-117,197-210, Part VII 494-519.

Hampson, G.F. (1908). The moths of India. Supplementary papers to the Volume in "Fauna of British India". Series III, pt. IX. Journal of the Bombay Natural History Society 18: 257-271, 572-582.

Hampson, G.F. (1912). The moths of India. Supplementary papers to the Volume in "Fauna of British India". Series IV, pt. V. Journal of the Bombay Natural History Society 21: 411-446, 878-911, 1222-1272.

Hampson, G.F. (1913a). Catalogue of the Lepidoptera-Phalanae British Museum 12, xiii + 626 pls. 192-221.

Hampson, G.F. (1913b). Catalogue of the Lepidoptera-Phalanae British Museum 13, xiv-609 pls. 222-239.

Hampson, G.F. (1924). Description of two new genera and two new species of Ophiderinae (Noctuidae). Entomologist 57: 182-184.

Hargreaves, E. (1936). Fruit - piercing Lepidoptera in Sierra Leone. Bulletin of Entomological Research 27: 589-605.

Holloway, J.D. (1977). The Lepidoptera of Norfolk Island, their Biogeography and Ecology. Series Entomologica 13, Junk: The Hague. Kalshoven, L.G.E. (1951). De plagen van de cultuurgewassen in Indonesië 2: 615-617. La Haye's Gravenhage.

Kapur, A.P. and G.S. Arora (1971). Taxonomic studies on some Indian species of the genus Agrotis Ochsenheimer and allied genera (Noctuidae: Lepidoptera). Records of Zoological Survey 65(1-4): 89-166.

Khoshoo, T.N. (1995). Census of India's Biodiversity: Tasks ahead. Current Science 69(1): 14-17.

Kirti, J.S. and H.S. Rose (1987). Taxonomic status of two north- 
eastern India species referred to genus Sylepta Hübner with the proposal of a new genus Hemopsis. Entomon 12(4): 379-383.

Kirti, J.S. and H.S. Rose (1989). Indogrammodes gen. nov. for Polygrammodes pectinicornalis (Guenée) (Pyraustinae: Pyralidae: Lepidoptera). Journal of the Bombay Natural History Society 86(3): 411-414.

Kirti, J.S. and H.S. Rose (1990). Taxonomic status of three Indian species of genus Botyodes Guenée (Pyraustinae: Pyralidae: Lepidoptera). Journal of Insect Science 3(2): 118-121.

Kirti, J.S and H.S. Rose (1992). Studies on Indian species of the genus Palpita Hübner (Lepidoptera: Pyralidae: Pyraustinae). Journal of Entomological Research 16(1): 62-77.

Kirti, J.S. and J.S. Sodhi (2001). A systematic list of Pyraustinae of northeastern India (Pyralidae: Lepidoptera). Zoos' Print Journal 16(10): 607-614.

Kitching, I.J. (1984). A historical review of the higher classification of the Noctuidae (Lepidoptera). Bulletin of British Museum and Natural History (Entomology) 49(3): 153-234.

Klima, A. (1939). Pyralidae. Pyraustinae, Lepidopteran Catalogue 89: 224 pp., 94: 225-384. S. Gravenhage.

Kothari, A. (2001). An exercise in Conservation. Frontline 18(2): $67-$ 71.

Mehta, D.R. (1933). Comparative morphology of the male genitalia in lepidoptera. Record of Indian Museum 35: 197-266, 114 figs.

MoEF (1998). Implementation of Article 6 of the Convention on Biological Diversity in India. National Report, 59 pp.

Moore, F. (1867). On the lepidopterous fauna of Bengal. Proceedings of Zoological Society of London pp. 44-98, 612-686.

Moore, F (1878). Description of new species of Lepidoptera collected by late F.Stoliczka during Indian Government Mission to Yarkland in 1873. Annual Magazine of Natural History 1(5):227-237.

Moore, F. (1881). On the genera and species of the Lepidopterous subfamily Ophiderinae inhabiting the Indian region. Transactions of the Zoological Society XI: 63-73, pls. XII-XIV.

Munroe, E. (1950). The generic position of some North American species commonly referred to Pyrausta Schrank (Lepidoptera: Pyralidae). Canadian Entomologist, 82: 217-231, 28 figs.

Munroe, E. (1952). The illibalis group of the genus Palpita Hübner (Lepidoptera: Pyralidae). Canadian Entomologist 84: 43-55, 32 figs. Munroe, E. (1954). A new Phlyctaenia with notes on two related species (Lepidoptera: Pyralidae). Canadian Entomologist 86: 428-431. Munroe, E. (1957). North American components of the genus Diasemia of authors (Lepidoptera: Pyralidae). Canadian Entomologist 89: 164170, 15 figs.

Munroe, E. (1959). New species and a new subspecies of Palpita (lepidoptera: Pyralidae). Canadian Entomologist 91: 641-650, 9 figs. Munroe, E. (1960). New species of Polygrammodes and a related new genus (Lepidoptera: Pyralidae).Canadian Entomologist 92: 279-284, 9 figs.

Nayar, K.K, T.N. Ananthakrishnan and B.V. David (1983). General and Applied Entomology $4^{\text {th }}$ Reprint, Tata McGraw-Hill Publishing Company Ltd., New Delhi, 589pp.

Nazmi, N.H. (1963). A redescription of the Pyraustinae of Egypt (UAR) (Lepidoptera). Bulletin of Society of Entomologists, Egypte Cario 47: 201-250, 26 figs.

Nye, I.W.B. (1975). The Generic Names of the Moths of the World 1: 568pp. (Trustees of the British Museum), London.

Ogata, M. (1954). Notes on the genus Hypocala from Japan (Lepidoptera, Phalaenidae). Tinea 1: 48-53, 7 figs.
Pajni, H.R. and H.S. Rose (1972). An unusual frenulum of Pycnarmon cribrata Fabricius (Lepidoptera: Pyraloidea). Current Science 41(1): 36-37.

Pajni, H.R. and H.S. Rose (1977). Male genitalia of family Pyraustidae (Lepidoptera: Pyralidoidea). Research Bulletin (Science) Punjabi University 28(3\&4): 131-141.

Pajni, H.R. and H.S. Rose (1978). Revision of the genus Pagyda Walker for the revalidation of its synonym Synclera Lederer along with the description of a new species (Lepidoptera: Pyralidoidea: Pyraustidae). Entomon 3(2): 215-219.

Pajni, H.R., J. Singh and H.S. Rose (1985). Studies on the genus Grammodes Guenée (Catocalinae: Noctuidae: Lepidoptera). Geobios New Reports 4: 29-33.

Pelletier, J. (1982). Revision of the genus Episparis (Lepidoptera, Noctuidae, Ophiderinae). Annals of the Society of Entomology, France 18(2): 229-286.

Pierce, F.N. (1909). The genitalia of the group Noctuidae of the Lepidoptera of British Islands, 88pp.

Pierce, F.N. (1942). The genitalia of the group Noctuidae of the Lepidoptera of the British Islands. An account of the morphology of the female reproductive organs. Oundle, Northants, $64 \mathrm{pp}$.

Pruthi, H.S. (1969). Textbook on Agricultural Entomology, ICAR, New Delhi. Job Press Pvt. Ltd. Kanpur, 977p.

Robinson, G.S., K.R. Tuck and M. Shaffer (1994). A field guide to the smaller moths of South- East Asia. Malaysian Nature Society, Kauala Lumpur, 308 pp.

Rose, H.S. (1982a). Male genitalia of the type-species of some Pyraustinae (Lepidoptera: Pyralidae) from North India and its taxonomic significance. Journal of Entomological Research 6(1): 51-67.

Rose, H.S. (1982b). Key to the type species of some Pyraustinae (Lepidoptera: Pyralidae). Journal of Biological Research 2(2): 105109.

Rose, H.S. and S.S. Dhillon (1980). Historical account of Indian Pyralidae (Lepidoptera) and future scope of work on this family. The Indian Zoologist 4(1\&2): 137-151.

Rose, H.S. and S.S. Dhillon (1982). Studies on the female genitalia of the type-species of North Indian Pyraustinae (Lepidoptera: Pyralidae) and their taxonomic significance. Journal of Biological Research 2(1): 11-20.

Rose, H.S. and N.J. Kaur (1990). Further notes on Alamis ubrina Guenée- A polymorphic species (Catocalinae: Noctuidae: Lepidoptera). Geobios News Reports 9: 8-10.

Rose, H.S. and J.S. Kirti (1986). Comments on the genus Bocchoris Moore along with the description of a new genus Nankogobinda (Pyraustinae: Pyralidae: Lepidoptera). Journal of Entomological Research 10(1): 63-69.

Rose, H.S. and J.S. Kirti (1987). A new genus and species of Pyraustinae (Pyralidae: Lepidoptera) from India. Journal of the Bombay Natural History Society 84(2): 392-395.

Rose,H.S. and J.S. Kirti (1989). First record of genus Toxobotys Munroe and Mutuura (Pyraustinae: Pyralidae: Lepidoptera) from the Oriental region. Journal of Insect Science 2(1): 10-13.

Rose, H.S. and H.R. Pajni (1979). The taxonomic position of three North-Western species commonly referred to the genus Pyrausta Schrank (Lepidoptera: Pyraustinae). Journal of New York Entomological Society 82(1): 2-8.

Rose, H.S. and A.P. Singh (1989). A new species of genus Patania Moore (Lepidoptera: Pyralidae: Pyraustinae). Entomon 14 (1\&2): 3943. 
Rose, H.S. and J. Singh (1985). Further studies on the Indian species of the genus Polythlipta Lederer (Pyraustinae: Pyralidae: Lepidoptera). Biologica 1(1): 53-59.

Rose, H.S. and A. Srivastava (1988). Further nates on the species Platyja umminia (Cramer)- A ploymorphic Ophiderine (Ophiderinae: Noctuidae: Lepidoptera). Journal of Entomological Research 12(1): 911.

Rose, H.S. and A. Srivastava (1989a). A new Lepidopteral Indocala gen. nov. from India (Ophiderinae: Noctuidae: Lepidoptera). Journal of the Bombay Natural History Society 86(3): 411-414.

Rose, H.S. and A. Srivastava (1989b). Comments on the Indian species of the genus Asticta Hübner (Lepidoptera). Geobios New Reports 8: 13-16.

Rose, H. S. and A. Srivastava (1994). Placement of the specis of genus Othreis Hübner hitherto described under Ophideres Boisduval (Ophiderinae: Noctuidae: Lepidoptera). Annals of Entomology 12(1): 87-93.

Sevastopulo, D.G. (1956). Notes on the Heterocera of Calcutta (Lepidoptera). Journal of the Bombay Natural History Society 54: 302 308.

Shull, E.M. and N.T. Nadkerny (1961). Collecting moths by a mercury vapour lamp in Surat Dangs, Gujrat State. Journal of the Bombay Natural History Society 61(2): 281-

Srivastava, A. (1992). Genitalic studies of two species of genus Hulodes. Journal of Insect Science 5(1): 66-67.

Srivastava, A. and H.S. Rose (1987). Hypocala Guenée-A homogeneous group (Ophiderinae: Noctuidae: Lepidoptera). Science and Environment 9(1\&2): 27-34.
Srivastava, A. and H.S. Rose (1989). Identification of Indian species of the genus Anomis Hübner (Ophiderinae: Noctuidae: Lepidoptera). Entomon 14(3 \& 4): 237-244.

Srivastava, A. and H.S. Rose (1990). Studies on Delgamma complex in India (Ophiderinae: Noctuidae: Lepidoptera). Ad. Bios. 1990: 85-90. Srivastava, A. and H.S. Rose (1993a). Studies on the genitalia of the type-species of some Ophiderines (Lepidoptera:Noctuidae). Journal of the Bombay Natural History Society 90(2): 213-222.

Srivastava, A. and H.S. Rose (1993b). Genitalic studies of some Ophiderine moths (Noctuidae: Lepidoptera) and their significance. Journal of Insect Science 6(2): 239-245.

Sugi, S. (1961). On the Japanese species of the genus Calpe Treit (Lep., Noctuidae). Kontyu 29: 94-105, 18 figs.

Swinhoe,C. (1889). On the new Indian Lepidoptera, chiefly Heterocera. Proceedings of Zoological Society of London 1889: 396-436.

Swinhoe,C. (1895). A list of Lepidoptera of Khasia Hills. Transactions of the Entomological Society of London 1894:1-75.

Wang, P.Y. (1963). Notes on the Chinese Pyralid moths of the genus Diaphania Hübner (Lepidoptera). Acta Entomology Sinica Peking 12: 358-36

Wileman, A. E. (1922). On a new species of genus Calpe from Japan. Entomologist 55: 197-199.

Wileman, A.E. and R.J. West (1928). Description of a new genus and new species of Noctuidae, subfamily Ophiderinae. Entomological Records of London 40: 174-176.

Wileman, A.E. and R. J. West (1929). Description of a new genus and species of Noctuidae, subfamily Ophiderinae. Entomological Records of London 41: 23-25, 47-50. 